\title{
Surface Plasmon-Enhanced Nanoantenna for Localized Fluorescence
}

\author{
Isa Kocakarin and Korkut Yegin \\ Department of Electrical and Electronics Engineering, Yeditepe University, 34755 Istanbul, Turkey \\ Correspondence should be addressed to Korkut Yegin, kyegin@yeditepe.edu.tr
}

Received 6 September 2012; Revised 5 December 2012; Accepted 6 December 2012

Academic Editor: Cheng Wei Qiu

Copyright ( $) 2012$ I. Kocakarin and K. Yegin. This is an open access article distributed under the Creative Commons Attribution License, which permits unrestricted use, distribution, and reproduction in any medium, provided the original work is properly cited.

Surface plasmon-enhanced gold nanoantenna structures on glass substrate are studied for increased localized electric field and fluorescence at the feed gap locations of the antennas. Dipole, Archimedean balanced spiral, and bowtie and double bowtie geometries are studied for surface plasmon effect. Different flare angles for bowtie geometries are compared to each other. Double bowtie geometry with dual polarization capability exhibited superior performance with almost $56 \mathrm{~dB}$ field enhancement factor. We also studied the effect of substrate thickness on electric field enhancement and we found that glass thickness plays a critical role for coherent addition of surface plasmons at the feed gap location. The surface plasmon effect is proven by considering perfect electric conductor model of gold instead of its modified Drude model.

\section{Introduction}

Optical antenna has emerged as a promising field for enhancing the efficiency of photo detectors, light emitters, biosensors, and spectroscopy [1-4]. With the advancements in key enabling technologies at nanoscale fabrication, these antennas have been built and proven to be useful for many applications at infrared and visible regions of electromagnetic spectrum [5-12]. One peculiarity that makes these antennas differ from traditional antennas at RF region is that they can utilize spontaneous emission to increase their emission rates [13-20]. Purcell [15], in his classical work, showed that a resonator coupled with the antenna structure can build up zero-point fluctuations within a small volume that can potentially increase the rate of optical transitions by several magnitudes of order. This idea has inspired the use of metal nanoparticles to excite surface plasmons to enhance localized emission rate. Although Purcell factors can be as large as $10^{5}$ for microcavities, the quality factors of plasmonic structures are usually less than 100 due to radiative and absorptive loss involved in the process of spontaneous emission.

With the advent of scanning near field optical microscopy and single molecule microscopy, experimental tools provided controlled laboratory experiments to examine the enhancement in fluorescence from single molecule in proximity to noble metal nanoparticles [21-27]. The underlying physics in the near-field interaction closely resembles the concept of optical antennas. Metal nanoparticles support surface Plasmon polariton modes that depend on the nanoparticle structural shape and its surroundings such as substrate underneath [28-32]. The interaction of these metal nanoparticles with the molecule in subwavelength range enhances spontaneous emission by several orders of magnitude.

Unlike its transmission and reception modes of operation, a nanoantenna can also serve as a good aid for fluorescence spectroscopy due to strong field enhancement localized in the feed region [33-41]. However, the shape and overall dimensions of the antenna with its substrate play a critical role in achieving local field enhancement while avoiding quenching. The structural shapes of optical antennas are quite similar to those of traditional antennas with one exception: ease of manufacturability. It is possible to propose many varieties of 2- and 2.5D planar traditional antennas to optical regime, but manufacturing tolerances and limits of processing technologies shorten the list to quite few simple structures. In that respect, rod and bowtie geometries have been preferred over other designs. Nevertheless, spirals and other structures have also been simulated for better performance.

We first study traditional antenna shapes for gold imprinted on glass substrate and compare their local field 
enhancement factors at the antenna feed gap. Instead of analyzing entire antenna surface, we only consider field enhancement at the feed location where a sample can be placed. We also study the effects of substrate dimensions on enhancement factors because excitation and coherent addition of surface wave plasmons at the feed gap are important. We prove that the local field (electric field) enhancement is due to the surface wave plasmon effect by comparing identical structure to that of a perfect electric conductor. Lastly, we propose polarization insensitive antenna structure for local field enhancement.

\section{Theoretical Background for Field Enhancement}

Using photokinetic rates at steady state, the fluorescence rate per molecule $F_{m}$ is given by [42]

$$
F_{m}=\kappa \phi \frac{\sigma I_{e}}{1+I_{e} / I_{\mathrm{sat}}}
$$

where $\kappa$ represents collection efficiency, $\phi$ is quantum efficiency $\left(\phi=\gamma_{\mathrm{rad}} /\left(\gamma_{\mathrm{rad}}+\gamma_{\mathrm{nonrad}}\right), \gamma_{\mathrm{rad}}\right.$ and $\gamma_{\text {nonrad }}$ are rate constants for radiative and nonradiative emission rates), $\sigma$ denotes excitation cross section, $I_{e}$ is the excitation intensity, and $I_{\text {sat }}$ is the saturation intensity. For $I_{e} \ll I_{\text {sat }}, F_{m} \sim \kappa \phi I_{e}$ and for $I_{e} \gg I_{\text {sat }}, F_{m}$ can be approximated as $F_{m} \sim \kappa \phi I_{\text {sat }} \approx$ $\kappa \gamma_{\text {rad }}$. Measured fluorescence $F_{m}(t)$ is expressed as

$$
F_{m}(t)=\int F_{m}(r) C(r, t) d r^{3}
$$

where $C(r, t)$ is the time-dependent concentration. When measured fluorescence is integrated over a period $T$, observed fluorescence can be stated as

$$
F=\int_{0}^{T} F_{m}(t) d t
$$

Single molecule radiative emission is proportional to molecule dipole moment and local excitation electric field: $\gamma_{\text {rad }} \propto|\vec{p} \cdot \vec{E}|^{2}$. Therefore, observed fluorescence is strongly dependent on local excitation electric field for the case where the excitation intensity is much larger than the saturation intensity.

To observe field enhancement due to spontaneous emission, one can consider metal on dielectric residing in $x$ $y$ plane with boundary normal pointing in $z$-direction. For infinite dimensions in transverse plane, one can solve Maxwell's equations for field components due to an incident plane wave excitation. It appears that only for transverse magnetic (TM) excitation surface wave plasmons can be excited if $\varepsilon_{d}>0, \varepsilon_{m}<0$, and $\varepsilon_{m}+\varepsilon_{d}<0$, where $\varepsilon_{d}$ and $\varepsilon_{m}$ are the permittivity of dielectric and metal, respectively. It can be easily shown that these conditions are equivalent to $\varepsilon_{m}^{\prime}<-\varepsilon_{d}$, where $\varepsilon_{m}=\varepsilon_{m}^{\prime}+i \varepsilon_{m}^{\prime \prime}$. There are only few noble metals ( $\mathrm{Au}, \mathrm{Ag}, \mathrm{Pt}$, etc.) that can satisfy these conditions for frequencies below plasma frequency. Thus, it is possible to excite surface plasmons and design the structure in a way that surface plasmons add coherently at a specified location for increased localized electric field and fluorescence.

\section{Antenna Design}

We consider glass substrate (Pyrex) with gold imprinted on it. Drude model of gold is used in the simulations and simulations were performed using CST microwave studio, a 3D electromagnetic field solver based on finite integration technique which is a modified version of finite-difference time domain (FDTD) solution technique. Drude model of gold, which is a modified free-electron gas model for metals in bound-electron region, has been extensively studied before and the relative dielectric constant of gold can be approximated as

$$
\varepsilon_{r}(\omega)=\varepsilon_{\infty}-\frac{\omega_{p}^{2}}{\omega^{2}+i \gamma \omega},
$$

where $\varepsilon_{\infty}, \omega_{p}$, and $\gamma$ are the relative dielectric constant at infinity, plasma frequency, and collusion frequency, respectively. The values of $\omega_{p}$ and $\gamma$ are $1.216 \times 10^{16} \mathrm{rad} / \mathrm{s}$ and $0.2 \times 10^{14} \mathrm{rad} / \mathrm{s}$, and they are consistent with bulk material data [43]. We considered dipole antenna, spiral antenna, bowtie antenna, and double-bowtie antenna for local field enhancement. The structures are shown in Figure 1.

All of the antennas are assumed to reside on $1200 \times$ $1200 \mathrm{~nm}$ glass substrate and the thickness of the glass substrate is $1100 \mathrm{~nm}$. The dipole one arm length and feed gap separation are taken as $125 \mathrm{~nm}$ and $30 \mathrm{~nm}$, respectively. The thickness of the gold imprint is $10 \mathrm{~nm}$. Antenna resonance ( $\lambda=\lambda_{0} / \sqrt{\varepsilon_{r}}, \varepsilon_{r}$ is relative permittivity of glass substrate), according to classical antenna theory, is expected to be around $242 \mathrm{THz}$. For spiral geometry, the feed gap separation is optimized to $20 \mathrm{~nm}$ and the thickness of the gold is $14 \mathrm{~nm}$. Arm width of the Archimedean balanced spiral is $20 \mathrm{~nm}$, and only one turn with $70 \mathrm{~nm}$ turn radius is considered. Bowtie antenna has been studied for various flare angles, particularly for $30^{\circ}, 60^{\circ}$, and $90^{\circ}$. Feed gap and width of the bowtie have been varied for different geometries, but the length of one arm is kept constant at $125 \mathrm{~nm}$. The thickness is again taken as $10 \mathrm{~nm}$. In all cases, unity strength plane wave incident from glass substrate side, that is, opposite side of antenna, is assumed. In addition, we observe field enhancement at the center location of the feed region for all antennas. Field enhancement outside this region may reach higher levels than this location, but our primary interest is in applications fluorescence at the center of feed region. All simulations are carried out within $100-400 \mathrm{THz}$ frequency band due to limited applicability of Drude model.

\section{Results and Discussion}

The structures are simulated to study electric field enhancement at feed gap location. Although past studies concentrated on overall field enhancement on any location in proximity to antenna structure, we are particularly interested in the feed gap location. The dipole antenna is simulated first for reference and the result is displayed in Figure 2 for a plane wave polarized in $x$-direction.

Maximum field enhancement occurs at $166.5 \mathrm{THz}$ with $150 \mathrm{~V} / \mathrm{m}$ electric field at the feed location. Then, Archimedean balanced spiral antenna is simulated and the electric 


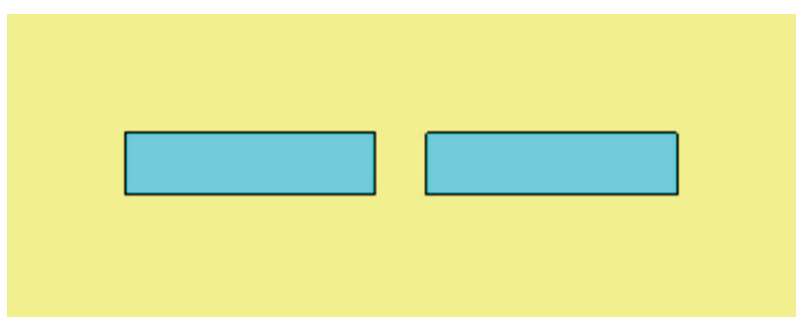

(a)

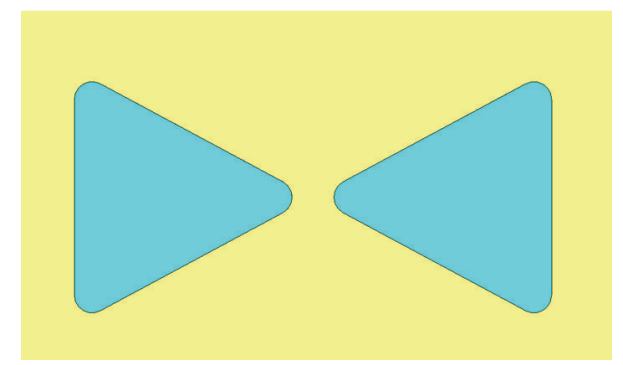

(c)

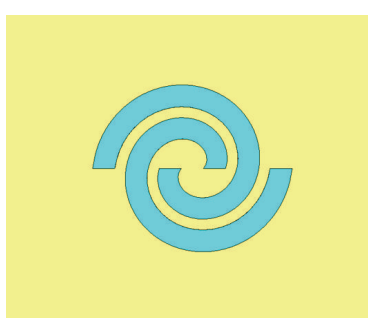

(b)

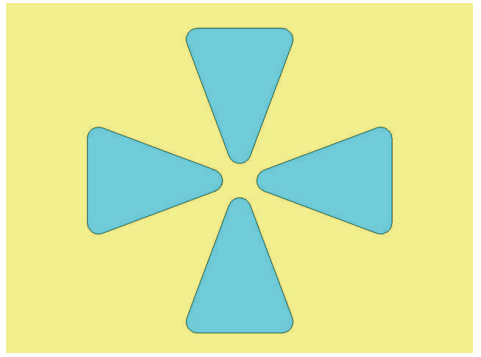

(d)

Figure 1: Nanoantenna structures considered for surface plasmon enhancement: (a) dipole, (b) Archimedean spiral, (c) bowtie, and (d) double bowtie.

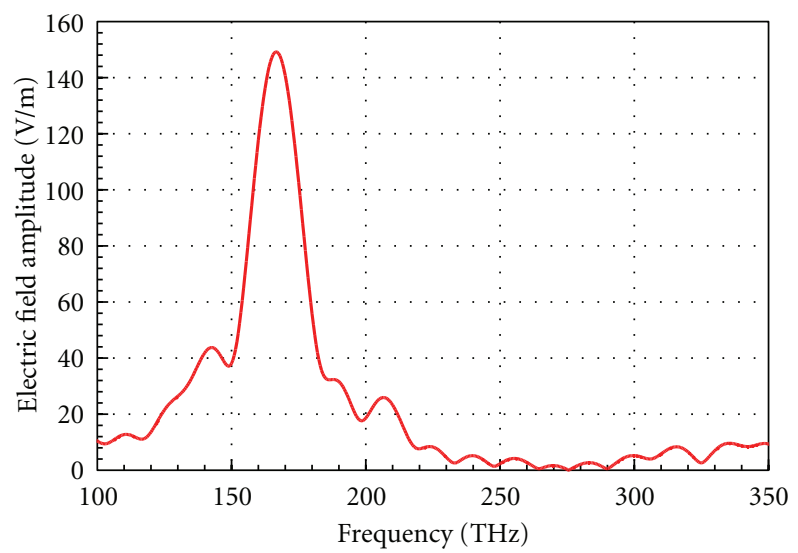

FIGURE 2: Electric field enhancement at the feed gap location of dipole antenna for $x$-polarized unity strength plane wave illumination.

field at the feed location is given in Figure 3. The spiral antenna is excited with $x$-polarized plane wave and the electric field components along $x$ - and $y$-directions are simulated.

It is observed that the electric field has a maximum at $184.5 \mathrm{THz}$ with $285 \mathrm{~V} / \mathrm{m}$ amplitude, which is 2.68 times larger than that of dipole antenna. The $y$-component of the electric field is about $92.5 \mathrm{~V} / \mathrm{m}$ at the same frequency. The field component along the straight edges of the spiral exhibits better field enhancement when it is excited with plane wave polarized in that direction. In our case, this is $x$-direction. When the antenna is excited with a $y$-polarized plane wave, field enhancement is lower. Thus, feed edge alignment greatly impacts field enhancement, and dual polarization capability of spiral is limited. The electric field distribution at $184 \mathrm{THz}$ is shown in Figure 4. We also note that we observe higher field value $(322 \mathrm{~V} / \mathrm{m})$ away from the center of the antenna.

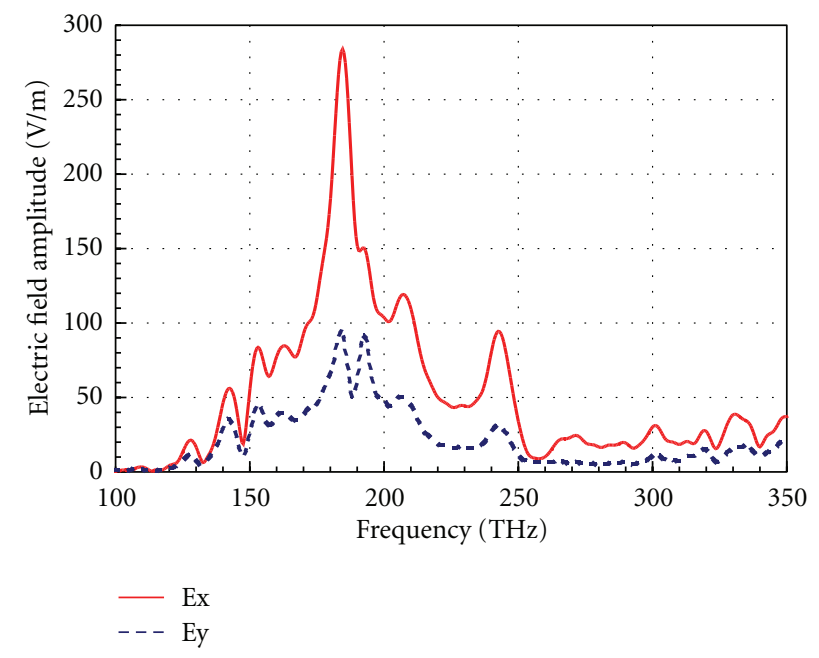

FIGURE 3: Electric field component along $x$ - and $y$-directions at the feed gap location of Archimedean spiral antenna for $x$-polarized unity strength plane wave illumination.

Next, bowtie antennas with $30^{\circ}, 60^{\circ}$, and $90^{\circ}$ flare angles are simulated, and the results are given in Figure 5 for $x$ polarized uniform plane wave illumination.

Electric field maximum occurs at $171 \mathrm{THz}$ with $174.5 \mathrm{~V} / \mathrm{m}$ for $30^{\circ}$ flare angle bowtie, at $183 \mathrm{THz}$ with $216 \mathrm{~V} / \mathrm{m}$ for $60^{\circ}$ flare angle bowtie, and at $172.5 \mathrm{THz}$ with $288 \mathrm{~V} / \mathrm{m}$ for $90^{\circ}$ flare angle bowtie. It is clear that $90^{\circ}$ bowtie outperforms other designs and it is 2.72 times larger than that of dipole antenna. It is well known in classical antenna theory that as flare angle is increased from $30^{\circ}$ to $90^{\circ}$, bowtie antenna possesses larger bandwidth but not considerably higher gain as the current distribution along incident polarization direction remains relatively 


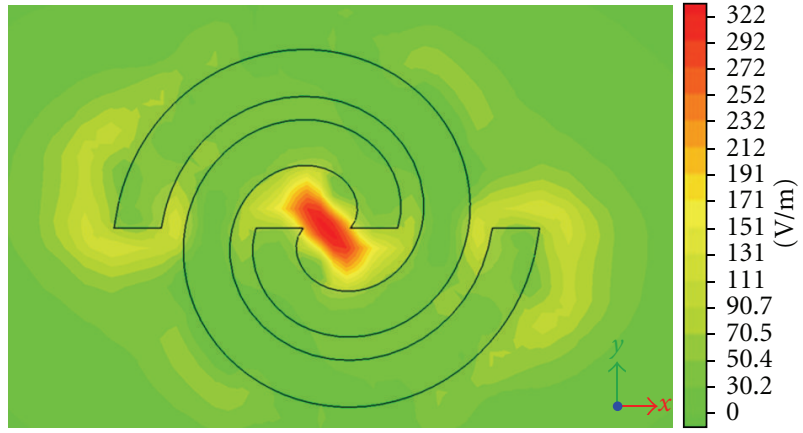

FIgURE 4: Electric field distribution on Archimedean spiral antenna at $184 \mathrm{THz}$ due to $x$-polarized unity strength plane wave incidence.

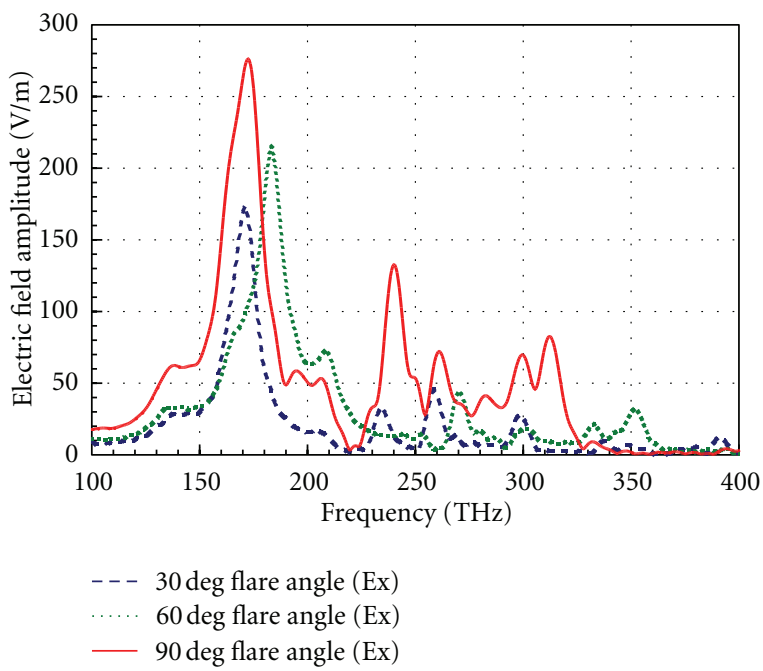

FIGURE 5: Electric field at the feed gap of bowtie with (a) $30^{\circ}$, (b) $60^{\circ}$, and (c) $90^{\circ}$ flare angles due to $x$-polarized unity strength plane wave incidence.

unchanged. However, with surface plasmon effect, field enhancement is considerably higher with $90^{\circ}$ flare angle bowtie. The electric field distribution on $90^{\circ}$ flare angle bowtie at $172.5 \mathrm{THz}$ is shown in Figure 6 .

To prove that electric field enhancement is indeed due to surface plasmon polariton mode, and we compared the electric field magnitudes of bowtie structure with a perfect electrical conductor (PEC); that is, the Drude model of gold metal is replaced with that of infinite conductivity. The electric field for PEC case is displayed in Figure 7. The electric field at $172.5 \mathrm{THz}$ for PEC model is 41, which is mostly due to first resonance of the structure.

Although bowtie provides a nice localized field enhancement, it is polarization sensitive. To overcome this shortcoming of bowtie, we studied double bowtie geometry for dual polarization. The simulation results for double bowtie geometries with different flare angles are shown in Figure 8. Surprisingly high value of double bowtie with $45^{\circ}$ flare angle is observed at the center of feed location. At $151 \mathrm{THz}$, field enhancement is 620 , which is more than 4 times of dipole antenna. As coupling within neighboring structures affect the frequency of maximum field enhancement, $90^{\circ}$

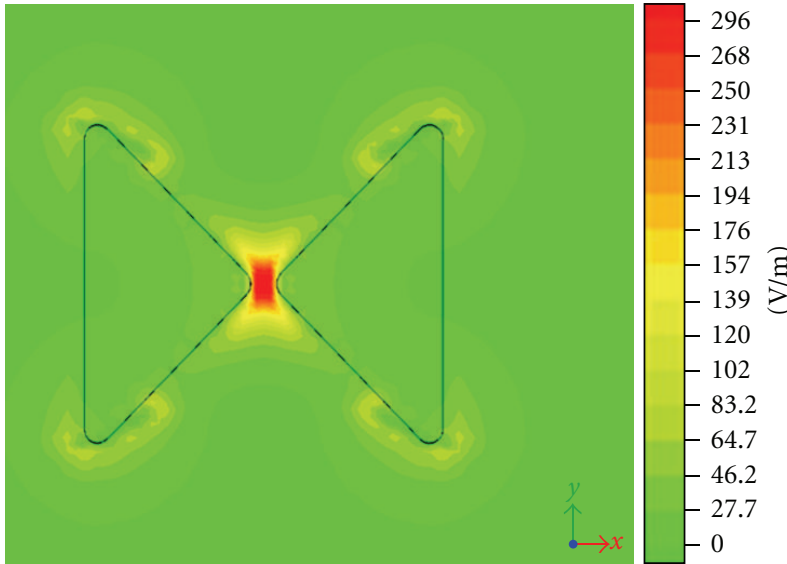

Figure 6: Electric field distribution on $90^{\circ}$ bowtie antenna at $172.5 \mathrm{THz}$ due to $x$-polarized plane wave incidence.

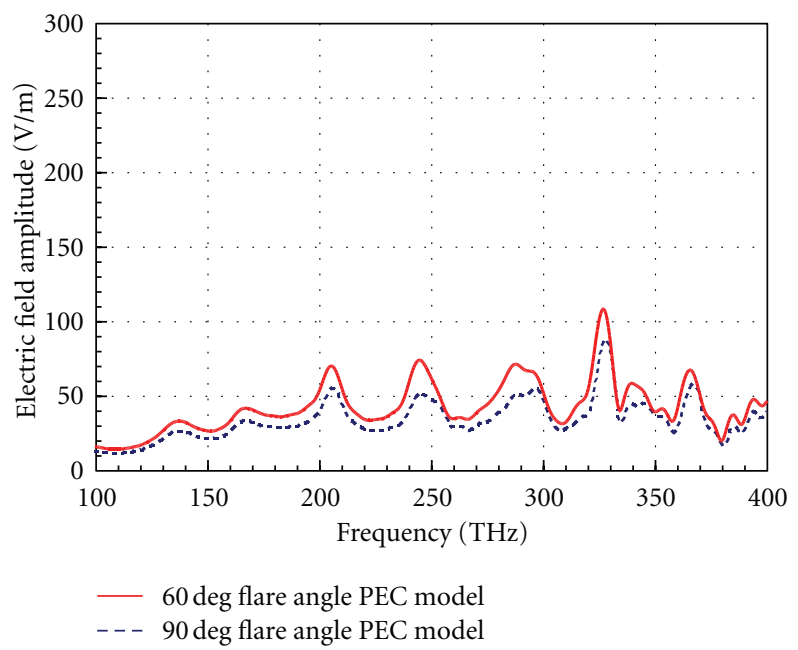

Figure 7: Electric field at the feed gap location of single bowtie for PEC model of gold.

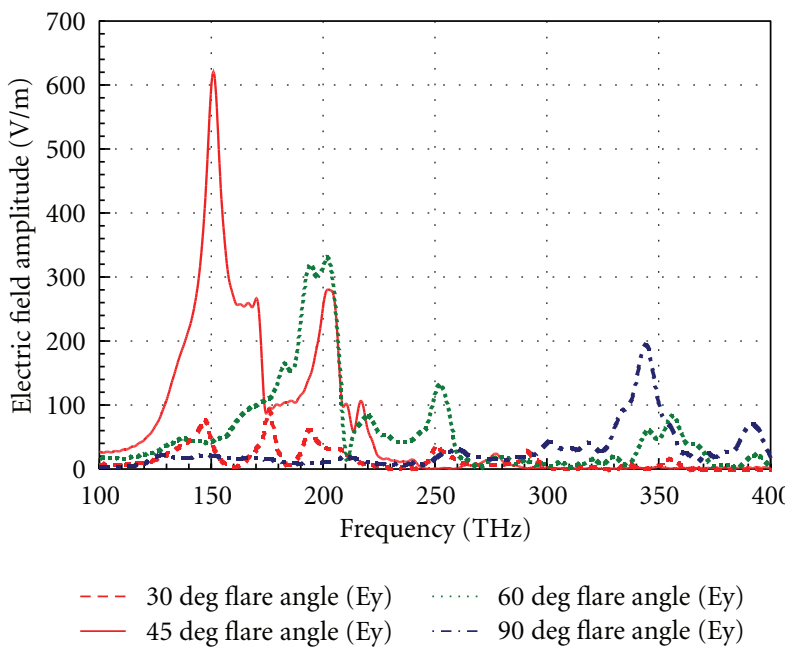

Figure 8: Electric field component along $y$-direction at the feed gap location of double bowtie antenna for $y$-polarized unity strength plane wave illumination. 
flare angle exhibited poor performance, but still higher than dipole antenna. When the structures are excited with $x$ polarized plane wave instead of $y$, same field enhancement factors are observed with very similar cross-polarization levels. Thus, double bowtie is indeed polarization insensitive compared to other structures and provided large enhancement factors. The electric field distribution on $45^{\circ}$ flare angle double bowtie at $151 \mathrm{THz}$ is shown in Figure 9. Electric field is confined to very small area compared to other structures and it exhibits very good localized field enhancement for numerous fluorescence applications.

To make sure that electric field enhancement is due to surface plasmon polariton mode, we again compared the electric field amplitudes of double bowtie structure with a perfect electrical conductor (PEC). The electric field for PEC case is displayed in Figure 10. The electric field for PEC model is at most $50 \mathrm{~V} / \mathrm{m}$, which is in line with single bowtie simulations.

We have also observed that glass thickness plays a critical role in field enhancement. As reflections from side walls and bottom side of the substrate may add up constructively and/or destructively at the feed location, one must carry out a thickness perturbation study on the electric field simulations. We varied substrate (glass) thickness from $1400 \mathrm{~nm}$ to $200 \mathrm{~nm}$ with $300 \mathrm{~nm}$ steps to observe the effect of thickness on electric field and the results are displayed in Figure 11. The frequency at which the electric field becomes maximum remains relatively unchanged as thickness varied. It is very difficult to calculate the resonances of the structure at these frequencies. If surface plasmon polariton effect is neglected, one can calculate dielectric rectangular cube resonances and perform a perturbation to account for metal presence on the structure. However, this is not possible in our case.

\section{Conclusions}

We have compared several nanoantenna structures for localized field enhancement, particularly at antenna feed gap location. We took several known structures and optimized their structural parameters to show that electric field enhancement is beyond the natural resonance of the antenna. We also proposed double bowtie antenna structure for dual polarization. Single bowtie antenna with $90^{\circ}$ flare angle exhibited promising field enhancement factor of about $50 \mathrm{~dB}$ above the incident plane wave illumination. To create relatively polarization insensitive structure, we proposed double bowtie structure and studied various flare angles for maximum field enhancement at the feed location. We achieved about $56 \mathrm{~dB}$ field enhancement with $45^{\circ}$ double bowtie structure. The structure is symmetric for dual polarization applications. Archimedean balanced spiral antenna also showed good performance, but manufacturing difficulties associated with this antenna precludes its realization compared to bowtie geometries. We studied the effects of substrate thickness on electric field enhancement and showed that substrate thickness can be very influential and must be taken into account during perturbational study of the antenna.

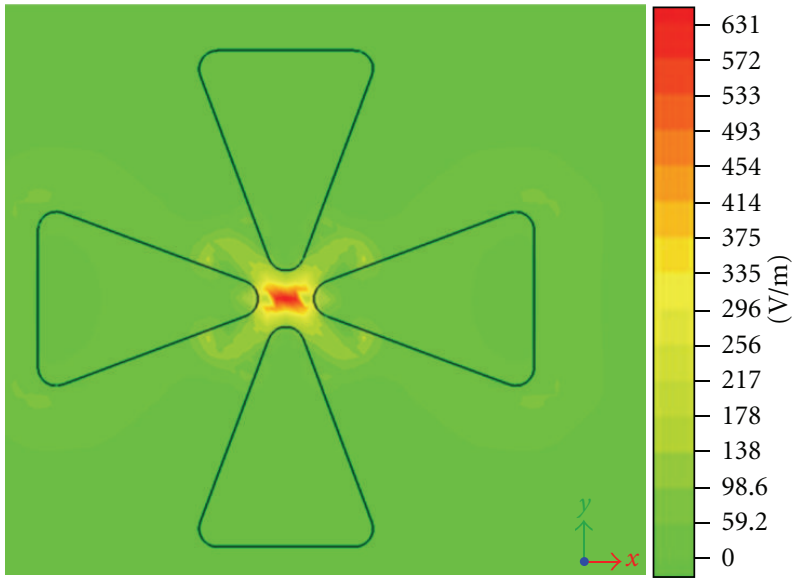

Figure 9: Electric field distribution on $45^{\circ}$ double bowtie antenna at $151 \mathrm{THz}$ due to $y$-polarized plane wave incidence.

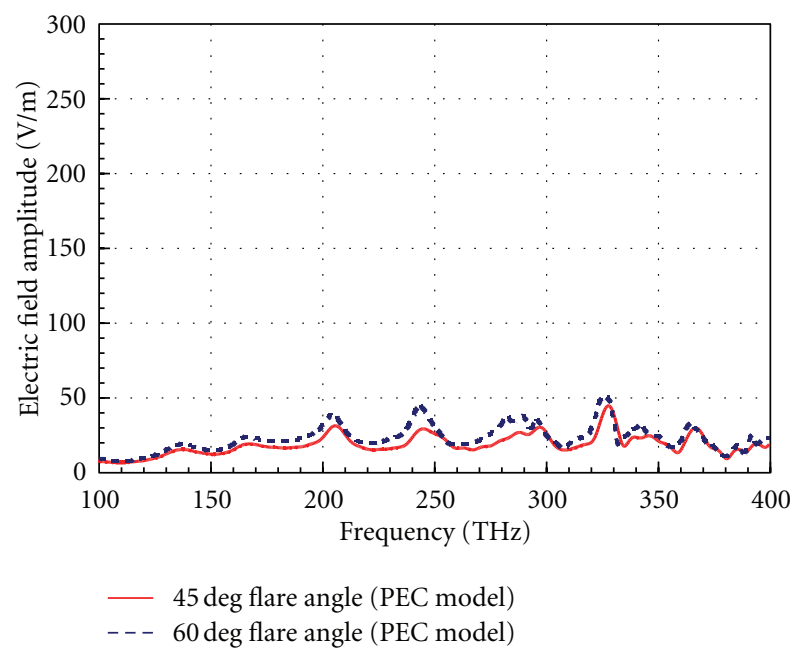

FIGURE 10: Electric field at the feed gap location of double bowtie for PEC model of gold.

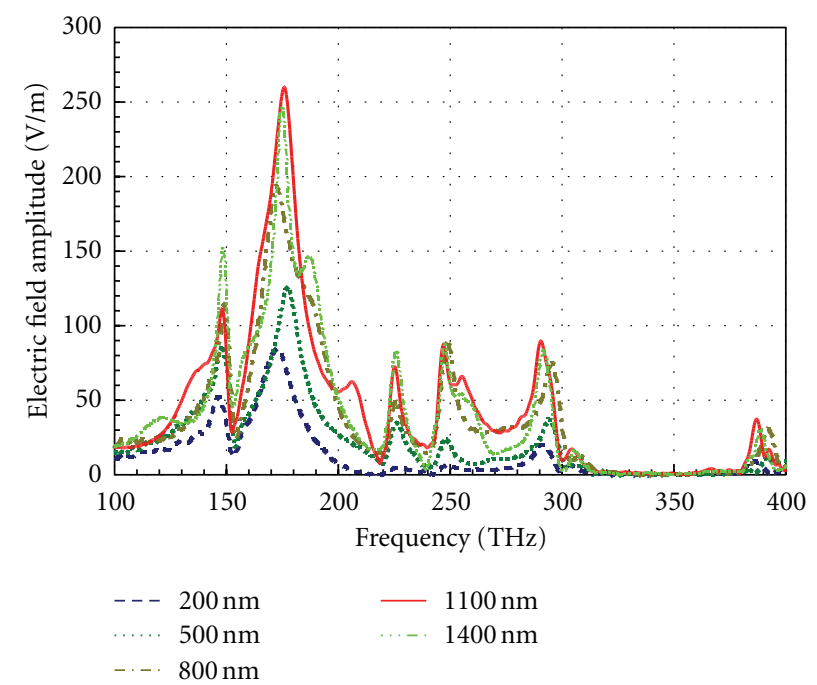

FIGURE 11: Electric field variation at the feed location of $90^{\circ}$ single bowtie for different glass thicknesses varying from $1400 \mathrm{~nm}$ to $200 \mathrm{~nm}$. 


\section{Acknowledgments}

The authors express thanks to the anonymous reviewers for their many helpful suggestions. This work is partly funded by Yeditepe University Research Fund.

\section{References}

[1] P. Muehlschlegel, H. J. Eisler, O. J. F. Martin, B. Hecht, and D. W. Pohl, "Resonant optical antennas," Science, vol. 308, pp. 1607-1609.

[2] P. Bharadwaj, B. Deutsch, and L. Novotny, "Optical antennas," Advances in Optics and Photonics, vol. 1, pp. 438-483, 2009.

[3] L. Novotny and N. F. van Hulst, "Antennas for light," Nature Photonics, vol. 5, no. 2, pp. 83-90, 2011.

[4] V. Giannini, A. I. Fernandez-Dominguez, S. C. Heck, and S. A. Maier, "Plasmonic nanoantennas: fundamentals and their use in controlling the radiative properties of nanoemitters," Chemical Reviews, vol. 111, pp. 3888-3912, 2011.

[5] L. Cao, J. S. Park, P. Fan, B. Clemens, and M. L. Brongersma, "Resonant germanium nanoantenna photodetectors," Nano Letters, vol. 10, no. 4, pp. 1229-1233, 2010.

[6] J. N. Farahani, D. W. Pohl, H. J. Eisler, and B. Hecht, "Single quantum dot coupled to a scanning optical antenna: a tunable superemitter," Physical Review Letters, vol. 95, no. 1, Article ID 017402, 4 pages, 2005.

[7] D. Gevaux, "Nano-antenna picks up green light," Nature Photonics, vol. 1, p. 90, 2007.

[8] L. Novotny, "Nano-optics: optical antennas tuned to pitch," Nature, vol. 455, no. 7215, p. 887, 2008.

[9] E. Ozbay, "Plasmonics: merging photonics and electronics at nanoscale dimensions," Science, vol. 311, no. 5758, pp. 189193, 2006.

[10] D. P. Fromm, A. Sundaramurthy, P. James Schuck, G. Kino, and W. E. Moerner, "Gap-dependent optical coupling of single "bowtie" nanoantennas resonant in the visible," Nano Letters, vol. 4, no. 5, pp. 957-961, 2004.

[11] L. Wang, S. M. Uppuluri, E. X. Jin, and X. Xu, "Nanolithography using high transmission nanoscale bowtie apertures," Nano Letters, vol. 6, no. 3, pp. 361-364, 2006.

[12] A. Sundaramurthy, P. J. Schuck, N. R. Conley, D. P. Fromm, G. S. Kino, and W. E. Moerner, "Toward nanometer-scale optical photolithography: utilizing the near-field of bowtie optical nanoantennas," Nano Letters, vol. 6, no. 3, pp. 355-360, 2006.

[13] P. Anger, P. Bharadwaj, and L. Novotny, "Enhancement and quenching of single-molecule fluorescence," Physical Review Letters, vol. 96, no. 11, Article ID 113002, 4 pages, 2006.

[14] G. Sun, J. B. Khurgin, and R. A. Soref, "Practicable enhancement of spontaneous emission using surface plasmons," Applied Physics Letters, vol. 90, no. 11, Article ID 111107, 2007.

[15] E. M. Purcell, "Spontaneous emission probabilities at radio frequencies," Physical Review, vol. 69, p. 681, 1946.

[16] R. R. Chance, A. Prock, and R. Silbey, "Molecular fuorescence and energy transfer near interfaces," Advances in Chemical Physics, vol. 37, p. 1, 1978.

[17] L. A. Blanco and F. J. García de Abajo, "Spontaneous light emission in complex nanostructures," Physical Review B, vol. 69, no. 20, Article ID 205414, 2004.

[18] R. G. Hulet, E. S. Hilfer, and D. Kleppner, "Inhibited spontaneous emission by a Rydberg atom," Physical Review Letters, vol. 55, no. 20, pp. 2137-2140, 1985.
[19] L. Rogobete, F. Kaminski, M. Agio, and V. Sandoghdar, "Design of plasmonic nanoantennae for enhancing spontaneous emission," Optics Letters, vol. 32, no. 12, pp. 1623-1625, 2007.

[20] A. Mohammadi, V. Sandoghdar, and M. Agio, "Gold nanorods and nanospheroids for enhancing spontaneous emission," New Journal of Physics, vol. 10, Article ID 105015, 2008.

[21] L. Novotny and S. J. Stranick, "Near-field optical microscopy and spectroscopy with pointed probes," Annual Review of Physical Chemistry, vol. 57, pp. 303-331, 2006.

[22] J. Wessel, "Surface-enhanced optical microscopy," Journal of the Optical Society of America B, vol. 2, pp. 1538-1540, 1985.

[23] H. G. Frey, S. Witt, K. Felderer, and R. Guckenberger, "Highresolution imaging of single fluorescent molecules with the optical near-field of a metal tip," Physical Review Letters, vol. 93, no. 20, Article ID 200801, 2004.

[24] K. B. Crozier, A. Sundaramurthy, G. S. Kino, and C. F. Quate, "Optical antennas: resonators for local field enhancement," Journal of Applied Physics, vol. 94, no. 7, pp. 4632-4642, 2003.

[25] W. L. Barnes, "Fluorescence near interfaces: the role of photonic mode density," Journal of Modern Optics, vol. 45, pp. 661-699, 1998.

[26] E. Fort and S. Gresillon, "Surface enhanced fluorescence," Journal of Physics D, vol. 41, Article ID 013001, 2008.

[27] J. Zhang, Y. Fu, M. H. Chowdhury, and J. R. Lakowicz, "Metalenhanced single-molecule fluorescence on silver particle monomer and dimer: coupling effect between metal particles," Nano Letters, vol. 7, no. 7, pp. 2101-2107, 2007.

[28] J. Enderlein and T. Ruckstuhl, "The efficiency of surface-plasmon coupled emission for sensitive fluorescence detection," Optics Express, vol. 13, no. 22, pp. 8855-8865, 2005.

[29] Y. J. Hung, I. I. Smolyaninov, C. C. Davis, and H. C. $\mathrm{Wu}$, "Fluorescence enhancement by surface gratings," Optics Express, vol. 14, no. 22, pp. 10825-10830, 2006.

[30] Y. Liu and S. Blair, "Fluorescence enhancement from an array of subwavelength metal apertures," Optics Letters, vol. 28, no. 7, pp. 507-509, 2003.

[31] F. Tam, G. P. Goodrich, B. R. Johnson, and N. J. Halas, "Plasmonic enhancement of molecular fluorescence," Nano Letters, vol. 7, pp. 496-501, 2007.

[32] R. M. Bakker, H. K. Yuan, Z. Liu et al., "Enhanced localized fluorescence in plasmonic nanoantennae," Applied Physics Letters, vol. 92, no. 4, Article ID 043101, 2008.

[33] A. Kinkhabwala, Z. Yu, S. Fan, Y. Avlasevich, K. Müllen, and W. E. Moerner, "Large single-molecule fluorescence enhancements produced by a bowtie nanoantenna," Nature Photonics, vol. 3, no. 11, pp. 654-657, 2009.

[34] L. Novotny, "Effective wavelength scaling for optical antennas," Physical Review Letters, vol. 98, Article ID 266802, 2007.

[35] J. Alda, J. M. Rico-García, J. M. López-Alonso, and G. Boreman, "Optical antennas for nano-photonic applications," Nanotechnology, vol. 16, no. 5, pp. S230-S234, 2005.

[36] F. Gonzalez and G. Boreman, "Comparison of dipole, bowtie, spiral and logperiodic IR antennas," Infrared Physics \& Technology Journal, vol. 146, pp. 418-428, 2004.

[37] R. D. Grober, R. J. Schoelkopf, and D. E. Prober, "Optical antenna: towards a unity efficiency near-field optical probe," Applied Physics Letters, vol. 70, no. 11, pp. 1354-1356, 1997.

[38] P. J. Burke, S. Li, and Z. Yu, "Quantitative theory of nanowire and nanotube antenna performance," IEEE Transactions on Nanotechnology, vol. 5, pp. 314-334, 2006.

[39] H. Fischer and O. J. F. Martin, "Engineering the optical response of plasmonic nanoantennas," Optics Express, vol. 16, no. 12, pp. 9144-9154, 2008. 
[40] M. L. Brongersma, "Plasmonics: engineering optical nanoantennas," Nature Photonics, vol. 2, no. 5, pp. 270-272, 2008.

[41] P. J. Schuck, D. P. Fromm, A. Sundaramurthy, G. S. Kino, and W. E. Moerner, "Improving the mismatch between light and nanoscale objects with gold bowtie nanoantennas," Physical Review Letters, vol. 94, no. 1, Article ID 017402, 4 pages, 2005.

[42] C. Zandler, J. Enderlein, and R. A. Keller, Eds., Single-Molecule Detection in Solution-Methods and Applications, Wiley-VCH, Berlin, Germany, 2002.

[43] P. B. Johnson and R. W. Christy, "Optical constants of the noble metals," Physical Review B, vol. 6, no. 12, pp. 4370-4379, 1972. 

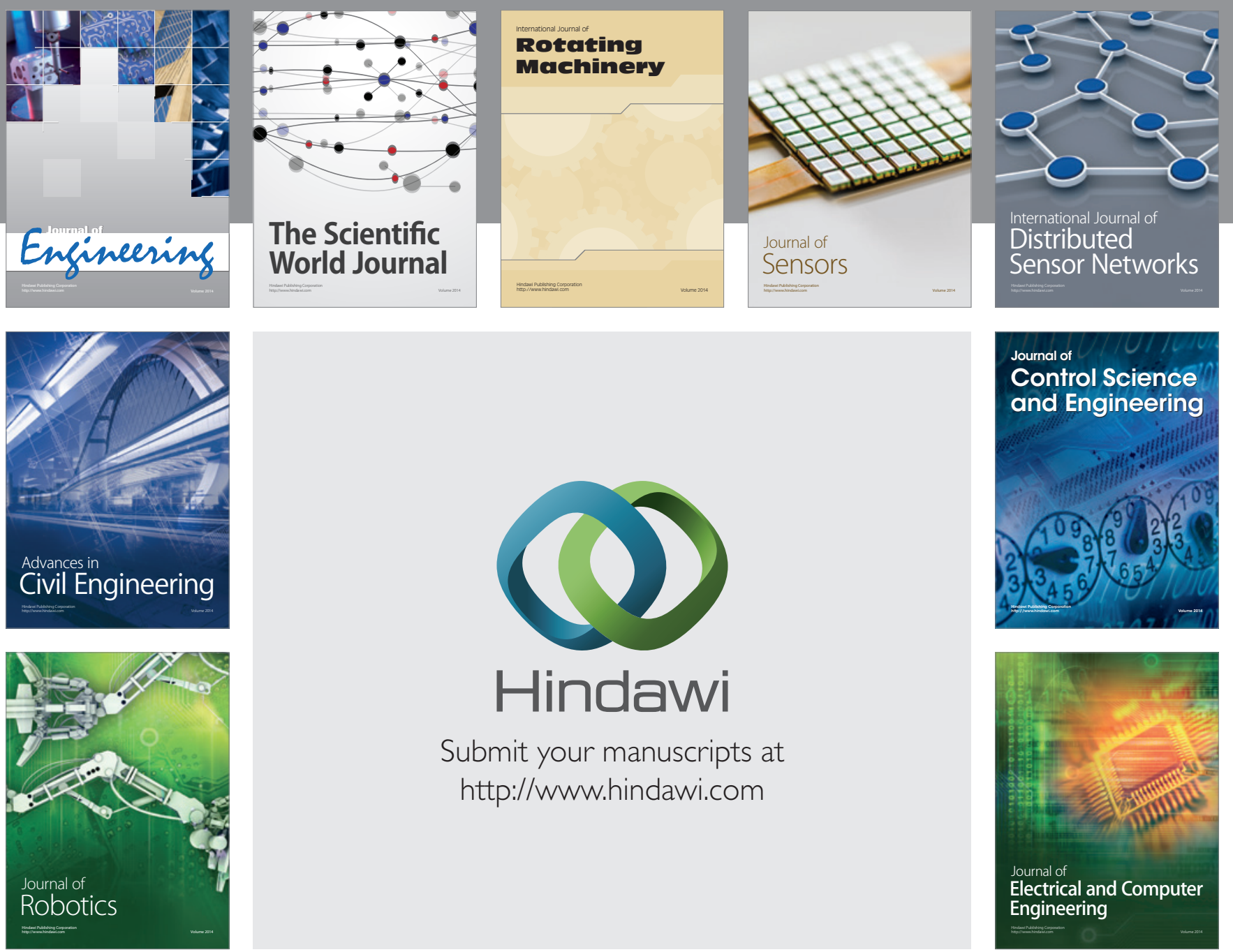

Submit your manuscripts at

http://www.hindawi.com
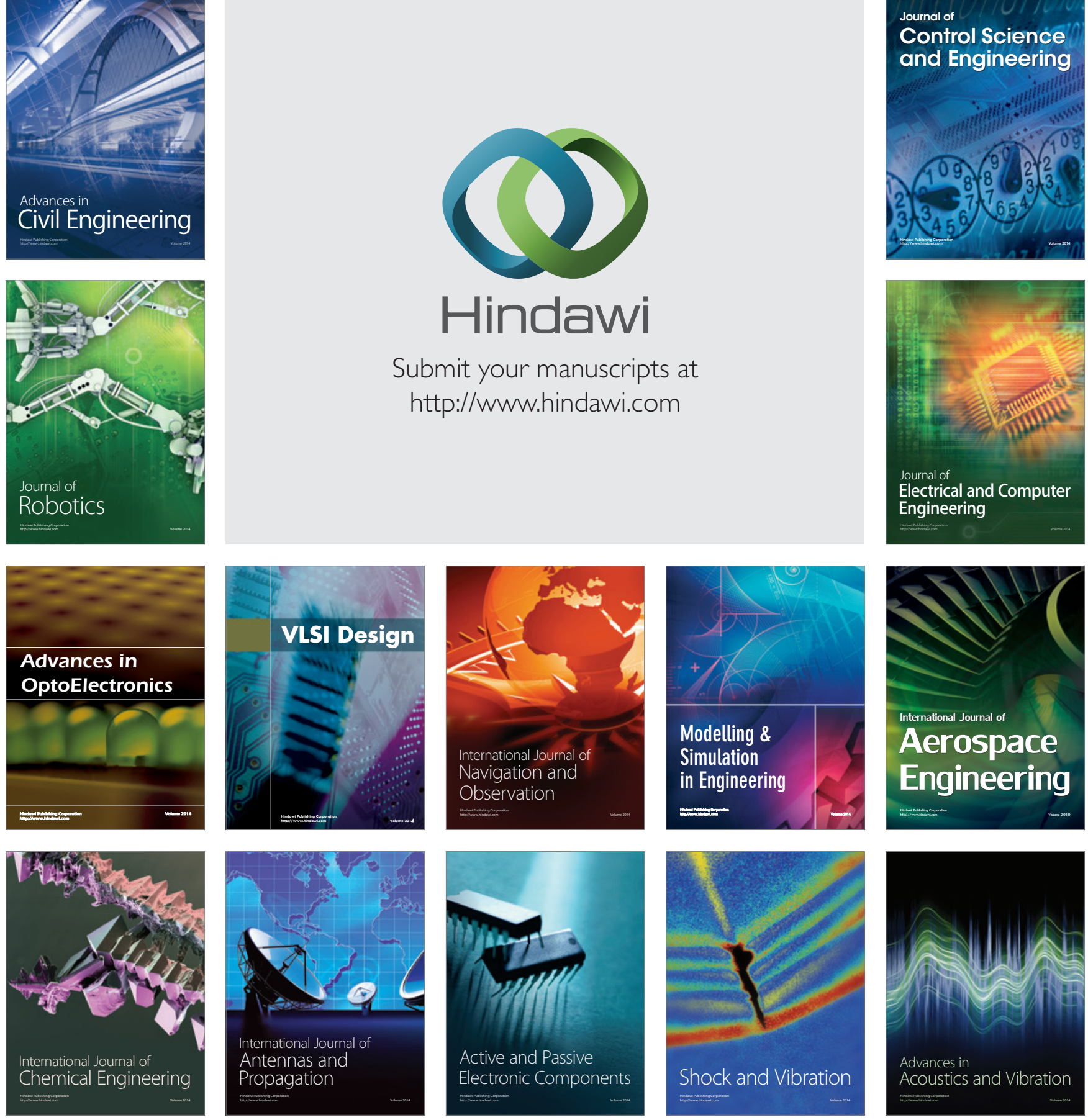